\title{
Discrete-Time Orthogonal Spline Collocation Method for One-Dimensional Sine-Gordon Equation
}

\author{
Xiaoquan Ding, ${ }^{1}$ Qing-Jiang Meng, ${ }^{2}$ and Li-Ping Yin ${ }^{3}$ \\ ${ }^{1}$ School of Mathematics and Statistics, Henan University of Science and Technology, Luoyang, Henan 471023, China \\ ${ }^{2}$ China Investment Securities, Shenzhen, Guangdong 518048, China \\ ${ }^{3}$ First Institute of Oceanography, State Oceanic Administration, Qingdao, Shandong 266061, China
}

Correspondence should be addressed to Qing-Jiang Meng; qjmeng04@163.com

Received 13 September 2015; Revised 23 November 2015; Accepted 1 December 2015

Academic Editor: Pilar R. Gordoa

Copyright (C) 2015 Xiaoquan Ding et al. This is an open access article distributed under the Creative Commons Attribution License, which permits unrestricted use, distribution, and reproduction in any medium, provided the original work is properly cited.

We present a discrete-time orthogonal spline collocation scheme for the one-dimensional sine-Gordon equation. This scheme uses Hermite basis functions to approximate the solution throughout the spatial domain on each time level. The convergence rate with order $\mathcal{O}\left(h^{4}+\tau^{2}\right)$ in $L^{2}$ norm and stability of the scheme are proved. Numerical results are presented and compared with analytical solutions to confirm the accuracy of the presented scheme.

\section{Introduction}

We consider the following one-dimensional sine-Gordon equation:

$$
\begin{aligned}
& \frac{\partial^{2} u}{\partial t^{2}}-\frac{\partial^{2} u}{\partial x^{2}}+F\left(u, u_{t}\right)=f(x, t), \\
& x \in\left(L_{0}, L_{1}\right), t>t_{0},
\end{aligned}
$$

with initial conditions

$$
\begin{aligned}
& u\left(x, t_{0}\right)=g(x), \\
&\left.\frac{\partial u}{\partial t}(x, t)\right|_{t=t_{0}}=\hat{g}(x), \\
& x \in\left[L_{0}, L_{1}\right]
\end{aligned}
$$

and Dirichlet boundary conditions

$$
\begin{aligned}
& u\left(L_{0}, t\right)=h_{0}(t), \\
& u\left(L_{1}, t\right)=h_{1}(t),
\end{aligned}
$$

$$
t \geq t_{0}
$$

or Neumann boundary conditions

$$
\begin{aligned}
& \frac{\partial u}{\partial x}\left(L_{0}, t\right)=\alpha(t), \\
& \frac{\partial u}{\partial x}\left(L_{1}, t\right)=\beta(t),
\end{aligned}
$$

$$
t \geq t_{0} .
$$

Here we require that $h_{0}\left(t_{0}\right)=g\left(L_{0}\right)$ and $h_{1}\left(t_{0}\right)=g\left(L_{1}\right)$ for consistency, $L_{0}<L_{1} \in \mathbb{R}$. When $F\left(u, u_{t}\right)=\sin (u)$ and $f(x, t)=0,(1)$ is a classical sine-Gordon equation. The sine-Gordon equation has applications in various research areas such as the Lie group of methods [1] and the inverse scattering transform [2]. It also appears in a number of other physical applications, including the propagation of fluxons in Josephson junctions between two superconductors, the motion of rigid pendulums attached to a stretched wire, and dislocations in crystals $[3,4]$.

The numerical solution to the sine-Gordon equation has received considerable attention in the literature. Among others Khaliq et al. [5] use a predictor-corrector scheme to solve the finite difference scheme using the methods of line. Bratsos [6] applies a predictor-corrector scheme from the use of rational approximation to the matrix-exponential term. Mohebbi and Dehghan [7] propose a high-order and accurate 
method for solving sine-Gordon equation using compact finite difference and DIRKN methods. Xu and Chang [8] present an implicit scheme and a compact scheme for the solution of an initial-boundary value problem of the generalized nonlinear sine-Gordon equation with a convergence rate $\mathcal{O}\left(\tau^{2}+h^{2}\right)$, where $h$ and $\tau$ denote the spatial and temporal mesh sizes, respectively. Cui [9] gives a three-level implicit compact difference scheme with a convergence rate $\mathcal{O}\left(\tau^{2}+h^{4}\right)$ by using the Padé approximant.

The purpose of this paper is to investigate the use of the orthogonal spline collocation (OSC) method with piecewise Hermite cubic polynomials for the spatial discretization of (1). The accuracy and stability of solutions with order $\mathcal{O}\left(\tau^{2}+\right.$ $\left.h^{4}\right)$ in $L^{2}$ norm are verified. This method has evolved as a valuable technique for the solution of many types of partial differential equations. See [10] for a comprehensive survey. The popularity of such a method is due in part to its conceptual simplicity and ease of implementation. One obvious advantage of the OSC method over the finite element method is that the calculation of the coefficient matrices is very efficient since no integral calculation is required. Another advantage of this method is that it systematically incorporates boundary conditions and interface conditions.

The paper is organized as follows. In Section 2, we briefly review the OSC method and give the discretization scheme of the sine-Gordon equation. In Section 3, we demonstrate the accuracy and stability of the scheme. Numerical results are presented in Section 4.

\section{The OSC Method for Sine-Gordon Equation}

With a positive integer $N$, let $\Delta$ be a partition of $\bar{\Omega}=\left[L_{0}, L_{1}\right]$ :

$$
\Delta: L_{0}=x_{0}<x_{1}<\cdots<x_{N}=L_{1} .
$$

Let $h_{j}=x_{j}-x_{j-1}, j=1,2, \ldots, N$, and $h=\max _{1 \leq j \leq N} h_{j}$. A family $\mathscr{F}$ of partitions is said to be quasi-uniform if there exists a finite positive number $\sigma$ such that

$$
\max _{1 \leq j \leq N} \frac{h}{h_{j}} \leq \sigma
$$

for every partition $\Delta$ in $\mathscr{F}$. We assume that the partition $\Delta$ is a member of a quasi-uniform family $\mathscr{F}$. Let $\left\{t_{n}\right\}_{n=0}^{J}$ be a partition of $[0, T]$, where $t_{n}=n \tau$ and $\tau=T / J$.

Let $\mathscr{M}$ be the space of piecewise Hermite cubics on $\Omega$ defined by

$$
\begin{aligned}
\mathscr{M}(\Delta)=\left\{v\left|v \in C^{1}(\bar{\Omega}): v\right|_{\left[x_{j-1}, x_{j}\right]} \in \mathscr{P}_{r}\right\}, & \\
& j=1,2, \ldots, N,
\end{aligned}
$$

where $\mathscr{P}_{r}$ denotes the set of all polynomials of degree less than or equal to $r$.

Let $\left\{\lambda_{k}\right\}_{k=1}^{2}$ denote the roots of the Legendre polynomial of degree 2 , where $\lambda_{1} \equiv(1 / 2)(1-1 / \sqrt{3})$ and $\lambda_{2} \equiv(1 / 2)(1+$ $1 / \sqrt{3})$. To apply the collocation method, we introduce a set of collocation points $\mathscr{G}=\left\{\xi_{j, k}\right\}_{j, k=1}^{N, 2}$ taken as

$$
\xi_{j, k}=x_{j-1}+h_{j} \lambda_{k}, \quad j=1,2, \ldots, N, k=1,2 .
$$

For $u, v \in C^{1}(\bar{\Omega})$, we define a discrete inner product and its induced norm by

$$
\begin{aligned}
\langle v, v\rangle_{\mathscr{G}} & =\sum_{j=1}^{N} h_{j} \sum_{k=1}^{2} \omega_{k} u\left(\xi_{j, k}\right) v\left(\xi_{j, k}\right), \\
\|u\|_{\mathscr{G}} & =\langle u, u\rangle_{\mathscr{G}}^{1 / 2} .
\end{aligned}
$$

We always use the following difference quotient notations:

$$
\begin{aligned}
& u_{t}^{n}=\frac{u^{n+1}-u^{n}}{\tau}, \\
& u_{\bar{t}}^{n}=\frac{u^{n}-u^{n-1}}{\tau}, \\
& u_{t \bar{t}}^{n}=\left(u_{t}^{n}\right)_{\bar{t}}, \\
& u_{\bar{t}}^{n}=\frac{u^{n+1}-u^{n-1}}{2 \tau} .
\end{aligned}
$$

Let $r$ be a nonnegative integer; we have

$$
\|u\|_{H^{r}(\Omega)}=\left(\sum_{j=0}^{r}\left\|\frac{\partial^{j} u}{\partial x^{j}}\right\|_{L^{2}(\Omega)}^{2}\right)^{1 / 2} .
$$

We denote by $L^{s}\left(0, T ; H^{r+3}(\Omega)\right)$ the Banach space of all $L^{s}$ integrable functions from $(0, T)$ into $H^{r+3}(\Omega)$ with norm

$$
\|u\|_{L^{s}\left(0, T ; H^{r+3}(\Omega)\right)}=\left(\int_{0}^{T}\|u\|_{H^{r+3}(\Omega)}^{s} d t\right)^{1 / s}
$$

for $s \in[1,+\infty)$ and the standard modification for $s=+\infty$. In this paper, we take $r=3$.

Let $\left\{\phi_{j}^{n}\right\}_{j=1}^{2 N}$ be basis functions of $\mathscr{M}(\Delta)$. So one may write

$$
u_{h}^{n}(x)=\sum_{j=1}^{2 N} \widehat{u}_{j}^{n} \phi_{j}^{n}(x), \quad n=0,1,2, \ldots, J
$$

where $\widehat{u}_{j}^{n}(j=1,2, \ldots, 2 N ; n=0,1,2, \ldots, J)$ are unknown coefficients which should be worked out.

We introduce the following lemmas.

Lemma 1 (Lemma 2.2 in [11], Equation 2.2 in [12]). For $u \in$ $M(\Delta)$, there exist positive constants $C_{1}$ and $C_{2}$ such that

$$
C_{1}\|u\|_{\mathscr{G}} \leq\|u\|_{L^{2}(\Omega)} \leq C_{2}\|u\|_{\mathscr{G}}
$$

Lemma 2 (Lemma 3.1, Lemma 3.2 in [11]). For $u, v \in \mathscr{M}(\Delta)$, one has

$$
\begin{aligned}
\left(u_{x}, v\right)_{\mathscr{G}} & =-\left(v_{x}, u\right)_{\mathscr{G}}, \\
\left(u_{x x}, v\right)_{\mathscr{G}} & =\left(u, v_{x x}\right)_{\mathscr{G}}, \\
-\left\langle u_{x x}, u\right\rangle_{\mathscr{G}} & \geq\left\|u_{x}\right\|_{L^{2}(\Omega)}^{2} .
\end{aligned}
$$


Lemma 3 (Theorem 4.1 in [11]). Let $u \in H^{6}(\Omega)$ and suppose that $W:[0, T] \rightarrow \mathscr{M}(\Delta)$ satisfies

$$
\begin{aligned}
\left(u_{x x}-W_{x x}\right)\left(\xi_{j, k}\right)-(u-W) & \left(\xi_{j, k}\right)=0, \\
j & =1,2, \ldots, N, k=1,2 .
\end{aligned}
$$

Then one has

$$
\|u-\Phi\|_{L^{2}(\Omega)} \leq C h^{4}\|u\|_{H^{6}(\Omega)} .
$$

Lemma 4 (Lemma 4 in [13]). Suppose that discrete function $w(n)$ satisfies the recurrence formula

$$
w_{n}-w_{n-1} \leq A \tau w_{n}+B \tau w_{n-1}+C_{n} \tau,
$$

where $A, B$, and $C_{n}(n=1, \ldots, N)$ are nonnegative constants. Then

$$
\max _{1 \leq n \leq N}\left|w_{n}\right| \leq\left(w_{0}+\tau \sum_{k=1}^{N} C_{k}\right) e^{2(A+B) T},
$$

where $\tau$ is small, such that $(A+B) \tau \leq(N-1) / 2 N(N>1)$.

Lemma 5 (Inequality (2.8) in [8]). Let $C_{1}, C_{2}$, and $C$ be constants. Suppose that the following conditions are satisfied:

(i) $F(x, y) \in C^{1}\left(\mathbb{R}^{2}\right)$;

(ii) $\left|F_{x}\right| \leq C_{1},\left|F_{y}\right| \leq C_{2}$.

Then one has

$$
|F(x, y)| \leq C(1+|x|+|y|) .
$$

We use finite difference scheme and construct the discrete-time OSC scheme as follows:

$$
\begin{aligned}
& {\left[\frac{1}{\tau^{2}}\left(u_{h}^{n+1}-2 u_{h}^{n}+u_{h}^{n-1}\right)-(1-2 \theta)\left(u_{h}^{n}\right)_{x x}\right.} \\
& \quad-\theta\left(\left(u_{h}^{n+1}\right)_{x x}+\left(u_{h}^{n-1}\right)_{x x}\right) \\
& \left.\quad+F\left(u_{h}^{n}, \frac{u_{h}^{n+1}-u_{h}^{n-1}}{2 \tau}\right)\right]\left(\xi_{j, k}\right)=f\left(\xi_{j, k}, t_{n}\right),
\end{aligned}
$$

for $n=1,2, \ldots, J, j=1,2, \ldots, N$, and $k=1,2$.

\section{Accuracy and Stability of the Scheme}

In this section, we study the accuracy and stability of the numerical method.

Theorem 6. Suppose $u(x, t) \in C^{2,4} \cap L^{2}\left(0, T ; H^{6}\right)$ is the solution of (21), $\partial u / \partial t, \partial^{2} u / \partial t^{2} \in L^{2}\left(0, T ; H^{6}\right)$, and $u_{h}^{n} \in$ $M(\Delta)(n=0,1, \ldots, J-1)$ is the solution of (16). If $W$ : $[0, T] \rightarrow \mathscr{M}(\Delta)$ is defined by $(16),\left\|\left(u_{h}^{0}-W^{0}\right)_{t}\right\|_{L^{2}(\Omega)}, \| u_{h}^{0}-$ $W^{0} \|_{H^{1}(\Omega)}$ and $\left\|u_{h}^{1}-W^{1}\right\|_{H^{2}(\Omega)}$ are $\mathcal{O}\left(\tau^{2}+h^{4}\right)$, then for $\tau$ and $h$ sufficiently small one has

$$
\max _{1 \leq n \leq J}\left\|u^{n}-u_{h}^{n}\right\|_{L^{2}(\Omega)}=\mathcal{O}\left(\tau^{2}+h^{4}\right),
$$

where $u^{n}(x)=u(x, n \tau)$ is the exact solution of (21) when $t=$ $n \tau$.
Proof. We use $C$ to denote a generic positive constant that is independent of $h$ and $\tau$ in the following proof. Substituting $u^{n}(x)=u(x, n \tau)$ into (21) and using Taylor expansion, we have

$$
\begin{aligned}
\frac{1}{\tau^{2}} & \left(u^{n+1}-2 u^{n}+u^{n-1}\right)-(1-2 \theta)\left(u^{n}\right)_{x x} \\
& -\theta\left(\left(u^{n+1}\right)_{x x}+\left(u^{n-1}\right)_{x x}\right)+F\left(u^{n},\left(u^{n}\right)_{\hat{t}}\right) \\
= & f\left(x, t_{n}\right)+\sigma^{n},
\end{aligned}
$$

where $\sigma^{n}=\mathcal{O}\left(\tau^{2}\right)$. Let $\hat{e}^{n}=u^{n}-W^{n}$ and $e^{n}=u_{h}^{n}-W^{n}$, then $u^{n}-u_{h}^{n}=\hat{e}^{n}-e^{n}$.

One may get from (21) and (23) that

$$
\begin{aligned}
& {\left[\frac{1}{\tau^{2}}\left(e^{n+1}-2 e^{n}+e^{n-1}\right)-(1-2 \theta) e_{x x}^{n}\right.} \\
& \left.\quad-\theta\left(e_{x x}^{n+1}+e_{x x}^{n-1}\right)\right]\left(\xi_{j, k}\right)=\left[\frac{1}{\tau^{2}}\left(\hat{e}^{n+1}-2 \hat{e}^{n}+\hat{e}^{n-1}\right)\right. \\
& \left.\quad-(1-2 \theta)\left(\hat{e}_{x x}^{n}\right)-\theta\left(\hat{e}_{x x}^{n+1}+\hat{e}_{x x}^{n-1}\right)\right]\left(\xi_{j, k}\right) \\
& \quad+F\left(u^{n},\left(u^{n}\right)_{\hat{t}}\right)-F\left(u_{h}^{n},\left(u_{h}^{n}\right)_{\hat{t}}\right)-\sigma^{n}\left(\xi_{j, k}\right) .
\end{aligned}
$$

Computing the inner product $\langle\cdot\rangle$ of (24) with $e^{n+1}-e^{n-1}$ as in Section 2, we have

$$
\begin{aligned}
\left\|e_{t}^{n}\right\|_{\mathscr{G}}^{2}-\left\|e_{t}^{n-1}\right\|_{\mathscr{G}}^{2}-(1-2 \theta)\left(e_{x x}^{n}, e^{n+1}-e^{n-1}\right) & \\
& -\theta\left(e_{x x}^{n+1}, e^{n+1}-e^{n-1}\right)-\theta\left(e_{x x}^{n-1}, e^{n+1}-e^{n-1}\right) \\
= & I_{1}+I_{2},
\end{aligned}
$$

where

$$
\begin{aligned}
I_{1} & =\left(\frac{1}{\tau^{2}}\left(\hat{e}^{n+1}-2 \hat{e}^{n}+\hat{e}^{n-1}\right)-(1-2 \theta) \hat{e}_{x x}^{n}-\theta\left(\hat{e}_{x x}^{n+1}\right.\right. \\
& \left.\left.+\hat{e}_{x x}^{n-1}\right)-\sigma^{n}, e^{n+1}-e^{n-1}\right)_{\mathscr{G}}=\tau \mid\left(\hat{e}_{t t}^{n}-(1-2 \theta) \hat{e}_{x x}^{n}\right. \\
& \left.-\theta\left(\hat{e}_{x x}^{n+1}+\hat{e}_{x x}^{n-1}\right)-\sigma^{n}, e_{t}^{n}+e_{t}^{n-1}\right)_{\mathscr{G}} \mid \leq C \tau\left(\left\|\hat{e}_{t t}^{n}\right\|_{\mathscr{G}}^{2}\right. \\
& +\left\|\hat{e}_{x x}^{n}\right\|_{\mathscr{G}}^{2}+\left\|\hat{e}_{x x}^{n+1}\right\|_{\mathscr{G}}^{2}+\left\|\hat{e}_{x x}^{n-1}\right\|_{\mathscr{G}}^{2}+\left\|\sigma^{n}\right\|_{\mathscr{G}}^{2}+\left\|e_{t}^{n}\right\|_{\mathscr{G}}^{2} \\
& \left.+\left\|e_{t}^{n-1}\right\|_{\mathscr{G}}^{2}\right), \\
I_{2} & =\left(F\left(u^{n},\left(u^{n}\right)_{\hat{t}}\right)-F\left(u_{h}^{n},\left(u_{h}^{n}\right)_{\hat{t}}\right), e^{n+1}-e^{n-1}\right) \\
& =\tau \sum_{j=1}^{N} h_{j} \sum_{k=1}^{r-1} \omega_{k}\left(F\left(u^{n},\left(u^{n}\right)_{\hat{t}}\right)-F\left(u_{h}^{n},\left(u_{h}^{n}\right)_{\hat{t}}\right)\right) \\
& \cdot\left(e_{t}^{n}+e_{t}^{n-1}\right) .
\end{aligned}
$$

Using Lemma 5, we can get

$$
\begin{aligned}
& \left|F\left(u^{n}, \frac{u^{n+1}-u^{n-1}}{2 \tau}\right)-F\left(u_{h}^{n}, \frac{u_{h}^{n+1}-u_{h}^{n-1}}{2 \tau}\right)\right| \\
& \quad \leq C\left[\left|e^{n}-e^{n}\right|+\frac{1}{2}\left(\left|\hat{e}_{t}^{n}\right|+\left|\hat{e}_{t}^{n-1}\right|+\left|e_{t}^{n}\right|+\left|e_{t}^{n-1}\right|\right)\right] .
\end{aligned}
$$


According to the definition of $W^{n}$, one can easily obtain $\hat{e}_{x x}^{n}=$ $\hat{e}^{n}$. Thus,

$$
\begin{aligned}
& \left\|e_{t}^{n}\right\|_{\mathscr{G}}^{2}-\left\|e_{t}^{n-1}\right\|_{\mathscr{G}}^{2}-(1-2 \theta)\left(e_{x x}^{n}, e^{n+1}-e^{n-1}\right) \\
& -\theta\left(e_{x x}^{n+1}, e^{n+1}-e^{n-1}\right)-\theta\left(e_{x x}^{n-1}, e^{n+1}-e^{n-1}\right) \\
& \leq C \tau\left(\left\|\hat{e}_{t t}^{n}\right\|_{\mathscr{G}}^{2}+\left\|\hat{e}_{t}^{n}\right\|_{\mathscr{G}}^{2}+\left\|\hat{e}_{t}^{n-1}\right\|_{\mathscr{G}}^{2}+\left\|\hat{e}^{n}\right\|_{\mathscr{G}}^{2}+\left\|\hat{e}^{n+1}\right\|_{\mathscr{G}}^{2}\right. \\
& +\left\|\hat{e}^{n-1}\right\|_{\mathscr{G}}^{2}+\left\|\sigma^{n}\right\|_{\mathscr{G}}^{2}+\left\|e_{t}^{n}\right\|_{\mathscr{G}}^{2}+\left\|e_{t}^{n-1}\right\|_{\mathscr{G}}^{2}+\left\|e^{n+1}\right\|_{\mathscr{G}}^{2} \\
& \left.+\left\|e^{n-1}\right\|_{\mathscr{G}}^{2}\right) .
\end{aligned}
$$

Applying Lemma 3, we have

$$
\begin{aligned}
& \left\|\hat{e}_{t}^{n}\right\|_{\mathscr{G}}=\left\|\frac{1}{\tau} \int_{0}^{\tau} \frac{\partial \widehat{e}}{\partial t}(n \tau+s) d s\right\|_{\mathscr{G}} \\
& \leq \frac{1}{\tau} \int_{0}^{\tau}\left\|\frac{\partial \widehat{e}}{\partial t}(n \tau+s)\right\|_{\mathscr{G}} d s \\
& \leq C_{1} h^{4}\left(\left\|\frac{\partial u}{\partial t}\right\|_{L^{\infty}\left(H^{6}\right)}+\|u\|_{L^{\infty}\left(H^{5}\right)}\right) \leq C_{2} h^{4}, \\
& \left\|\hat{e}_{t t}^{n}\right\|_{\mathscr{G}}=\left\|\frac{1}{\tau^{2}} \int_{-\tau}^{\tau}(\tau-|s|) \frac{\partial^{2} \widehat{e}}{\partial t^{2}}(n \tau+s) d s\right\|_{\mathscr{G}} \\
& \leq \frac{1}{\tau} \int_{-\tau}^{\tau}\left\|\frac{\partial^{2} \widehat{e}}{\partial t^{2}}(n \tau+s)\right\|_{\mathscr{G}} d s \\
& \leq C_{3} h^{4}\left(\left\|\frac{\partial^{2} u}{\partial t^{2}}\right\|_{L^{\infty}\left(H^{6}\right)}+\left\|\frac{\partial u}{\partial t}\right\|_{L^{\infty}\left(H^{5}\right)}+\|u\|_{L^{\infty}\left(H^{5}\right)}\right) \\
& \leq C_{4} h^{4},
\end{aligned}
$$

where $C_{i}, i=1, \ldots, 4$, denote constants. It follows from Lemma 1 and (28)-(29) that

$$
\begin{gathered}
\left\|e_{t}^{n}\right\|_{\mathscr{G}}^{2}-\left\|e_{t}^{n-1}\right\|_{\mathscr{G}}^{2}+\left\|e^{n+1}\right\|_{\mathscr{G}}^{2}-\left\|e^{n}\right\|_{\mathscr{G}}^{2}-(1-2 \theta) \\
\cdot\left(e_{x x}^{n}, e^{n+1}-e^{n-1}\right)-\theta\left(e_{x x}^{n+1}, e^{n+1}-e^{n-1}\right) \\
-\theta\left(e_{x x}^{n-1}, e^{n+1}-e^{n-1}\right) \leq C \tau\left[\left\|e^{n+1}\right\|_{\mathscr{G}}^{2}+\left\|e^{n-1}\right\|_{\mathscr{G}}^{2}\right. \\
\left.+\left\|e_{t}^{n}\right\|_{\mathscr{G}}^{2}+\left\|e_{t}^{n-1}\right\|_{\mathscr{G}}^{2}+\mathcal{O}\left(\tau^{2}+h^{4}\right)^{2}\right] .
\end{gathered}
$$

If $\theta>1 / 2$, by using similar arguments in the proof of Theorem 4.1 in [14], we have

$$
\begin{aligned}
\left\|e_{t}^{n}\right\|_{\mathscr{G}}^{2}-(1-2 \theta)\left(e_{x x}^{n+1}, e^{n}\right)_{\mathscr{G}}-\theta\left(e_{x x}^{n+1}, e^{n+1}\right)_{\mathscr{G}} \\
-\theta\left(e_{x x}^{n-1}, e^{n+1}\right)_{\mathscr{G}} \geq\left\|e_{t}^{n}\right\|_{\mathscr{G}}^{2} \\
-\frac{1}{2}\left[\left(e_{x x}^{n+1}, e^{n+1}\right)_{\mathscr{G}}+\left(e_{x x}^{n-1}, e^{n+1}\right)_{\mathscr{G}}\right] \geq\left\|e_{t}^{n}\right\|_{\mathscr{G}}^{2} \\
\quad+\frac{1}{2}\left(\left\|e_{x}^{n+1}\right\|_{\mathscr{G}}^{2}+\left\|e_{x}^{n}\right\|_{\mathscr{G}}^{2}\right) \geq\left\|e_{t}^{n}\right\|_{\mathscr{G}}^{2} \geq 0 .
\end{aligned}
$$

If $1 / 4 \leq \theta \leq 1 / 2$, then

$$
\begin{aligned}
& \left\|e_{t}^{n}\right\|_{\mathscr{G}}^{2}-(1-2 \theta)\left(e_{x x}^{n+1}, e^{n}\right)_{\mathscr{G}}-\theta\left(e_{x x}^{n+1}, e^{n+1}\right)_{\mathscr{G}} \\
& \quad-\theta\left(e_{x x}^{n-1}, e^{n+1}\right)_{\mathscr{G}} \geq\left\|e_{t}^{n}\right\|_{\mathscr{G}}^{2} \\
& \quad+\frac{1}{2}(4 \theta-1)\left(\left\|e_{x}^{n+1}\right\|_{\mathscr{G}}^{2}+\left\|e_{x}^{n}\right\|_{\mathscr{G}}^{2}\right) \geq\left\|e_{t}^{n}\right\|_{\mathscr{G}}^{2} \geq 0 .
\end{aligned}
$$

If $0<\theta<1 / 4$, by using Sobolev's inequality and Theorem 4.1 in [15], we have

$$
\begin{aligned}
& \left\|e_{t}^{n}\right\|_{\mathscr{G}}^{2}-(1-2 \theta)\left(e_{x x}^{n+1}, e^{n}\right)_{\mathscr{G}}-\theta\left(e_{x x}^{n+1}, e^{n+1}\right)_{\mathscr{G}} \\
& \quad-\theta\left(e_{x x}^{n-1}, e^{n+1}\right)_{\mathscr{G}} \geq\left\|e_{t}^{n}\right\|_{\mathscr{G}}^{2}-(1-2 \theta)\left(e_{x x}^{n+1}, e^{n}\right)_{\mathscr{G}} \\
& \quad \geq \frac{\alpha}{2}\left\|e_{t}^{n}\right\|_{\mathscr{G}}^{2} \geq 0,
\end{aligned}
$$

where $\alpha$ is a positive constant.

Thus, one can obtain from (30)-(33) that

$$
\omega^{n}-\omega^{n-1} \leq \tau\left(\omega^{n}+\omega^{n-1}\right)+C \tau\left(\tau^{2}+h^{4}\right)^{2},
$$

where

$$
\begin{aligned}
\omega^{n}= & \left\|e_{t}^{n}\right\|_{\mathscr{G}}^{2}+\left\|e^{n+1}\right\|_{\mathscr{G}}^{2}-(1-2 \theta)\left(e_{x x}^{n+1}, e^{n}\right)_{\mathscr{G}} \\
& -\theta\left[\left(e_{x x}^{n+1}, e^{n+1}\right)_{\mathscr{G}}+\left(e_{x x}^{n}, e^{n}\right)_{\mathscr{G}}\right] .
\end{aligned}
$$

Apply Lemma 4; after simple calculation we get the following inequality:

$$
\begin{aligned}
& \max _{1 \leq n \leq J-1} \omega^{n} \\
& \quad \leq\left(\omega^{0}+\tau \sum_{k=1}^{J-1} C_{5}\left(\tau^{2}+h^{4}\right)^{2}\right) \exp \left(C_{6}(J-1) \tau\right) \\
& \quad \leq C_{7}\left[\omega^{0}+\left(\tau^{2}+h^{4}\right)^{2}\right],
\end{aligned}
$$

where $C_{5}, C_{6}$, and $C_{7}$ denote constants.

Since $\theta>0$, we conclude

$$
\begin{aligned}
& \max _{1 \leq n \leq J-1}\left\{\left\|e^{n+1}\right\|_{\mathscr{G}}^{2}+\left\|e_{x}^{n+1}\right\|_{\mathscr{G}}^{2}+\left\|e^{n}\right\|_{\mathscr{G}}^{2}+\left\|e_{x}^{n}\right\|_{\mathscr{G}}^{2}\right\} \\
& \quad \leq C\left(h^{8}+\tau^{4}\right) .
\end{aligned}
$$

This implies

$$
\max _{1 \leq n \leq J}\left\|e^{n}\right\|_{\mathscr{G}} \leq C\left(h^{4}+\tau^{2}\right) .
$$

These all together yield the following inequality:

$$
\max _{1 \leq n \leq J}\left\|u^{n}-u_{h}^{n}\right\|_{L^{2}(\Omega)} \leq C\left(h^{4}+\tau^{2}\right)=C\left(h^{4}+\tau^{2}\right) .
$$

In the following theorem, we give the stability of the numerical method. 
Theorem 7. If the conditions of Theorem 6 are satisfied, then scheme (21) is unconditionally stable.

Proof. Let $\eta^{n}(x)$ be the error of $u_{h}^{n}(x)$ and $\widetilde{u}_{h}^{n}=u_{h}^{n}(x)-\eta^{n}(x)$. Then we have

$$
\begin{aligned}
& {\left[\frac{1}{\tau^{2}}\left(\eta^{n+1}-2 \eta^{n}+\eta^{n-1}\right)-(1-2 \theta) \eta_{x x}^{n}\right.} \\
& \left.\quad-\theta\left(\eta_{x x}^{n+1}+\eta_{x x}^{n-1}\right)\right]\left(\xi_{j, k}\right)=\left[F\left(\widetilde{u}_{h}^{n},\left(\widetilde{u}_{h}^{n}\right)_{\hat{t}}\right)\right. \\
& \left.\quad-F\left(u_{h}^{n},\left(u_{h}^{n}\right)_{\hat{t}}\right)\right]\left(\xi_{j, k}\right) .
\end{aligned}
$$

Computing the inner product of (40) with $\left(\eta^{n+1}-\eta^{n-1}\right)$, we obtain by a similar proof as that of Theorem 6 :

$$
\max _{1 \leq n \leq J}\left\|\eta^{n}\right\|_{\mathscr{G}} \leq C \widetilde{\omega}^{0}
$$

where

$$
\begin{aligned}
\widetilde{\omega}^{0}= & \left\|\eta_{t}^{0}\right\|_{\mathscr{G}}^{2}+\left\|\eta^{1}\right\|_{\mathscr{G}}^{2}+\left\|\eta^{0}\right\|_{\mathscr{G}}^{2}-(1-2 \theta)\left(\eta_{x x}^{1}, \eta^{0}\right)_{\mathscr{G}} \\
& -\theta\left(\eta_{x x}^{1}, \eta^{1}\right)_{\mathscr{G}}-\theta\left(\eta_{x x}^{0}, \eta^{0}\right)_{\mathscr{G}}
\end{aligned}
$$

According to [16] and references therein, this theorem expresses the generalized stability of the numerical scheme.

\section{Numerical Experiments}

In this section, we present some numerical results of our scheme for sine-Gordon equations. We adopt the following form of (1) for Examples 1 and 2:

$$
\frac{\partial^{2} u}{\partial t^{2}}+\frac{\partial u}{\partial t}-\frac{\partial^{2} u}{\partial x^{2}}+2 \sin (u)=f(x, t)
$$

According to (21), the corresponding OSC scheme might be written as

$$
\begin{aligned}
& \frac{1}{\tau^{2}}\left(u_{h}^{n+1}-2 u_{h}^{n}+u_{h}^{n-1}\right)+\frac{u_{h}^{n+1}-u_{h}^{n-1}}{2 \tau} \\
& \quad-(1-2 \theta)\left(u_{h}^{n}\right)_{x x}-\theta\left(\left(u_{h}^{n+1}\right)_{x x}+\left(u_{h}^{n-1}\right)_{x x}\right) \\
& \quad+2 \sin \left(u_{h}^{n}\right)=f(x, t)
\end{aligned}
$$

for $j=1,2, \ldots, N, n=0,1,2, \ldots, J-1$, and $k=1,2$.

Setting $\vec{\varphi}^{n}=\left[\widehat{\varphi}_{1}^{n}, \widehat{\varphi}_{2}^{n}, \ldots, \widehat{\varphi}_{2 N}^{n}\right]^{T}$ and substituting (13) into (44), one can obtain

$$
A\left(\vec{\varphi}^{n+1}\right)=B\left(\vec{\varphi}^{n}\right)+C\left(\vec{\varphi}^{n-1}\right)+D
$$

where $A, B, C$, and $D$ are matrices with special structures commonly known as almost block diagonal, so the system of algebraic equations (45) could be solved by using the COLROW algorithm [17].
Applying Taylor's theorem, one can get from (2) and (43)

$$
\begin{aligned}
u(x, \tau)= & z(x)+\mathcal{O}\left(\tau^{3}\right) \\
z(x)= & u_{0}(t)+\tau u_{1}(t) \\
& +\frac{\tau^{2}}{2}\left[\frac{\partial^{2} u_{0}}{\partial x^{2}}-\frac{\partial u_{1}}{\partial t}-2 \sin \left(u_{0}\right)\right](x) \\
& -\frac{\tau^{2}}{2} f(x, t) .
\end{aligned}
$$

Consequently, $u_{h}^{0}$ and $u_{h}^{1}$ can be prescribed by approximating $u_{0}(t)$ and $z(x)$ using piecewise Hermite cubic interpolations, respectively. In all of the following experiments, we choose $\theta=1 / 4$.

Example 1. We consider Dirichlet boundary conditions problem given in [9]. We consider the problem

$$
\begin{aligned}
\frac{\partial^{2} u}{\partial t^{2}}+ & \frac{\partial u}{\partial t}-\frac{\partial u^{2}}{\partial x^{2}}+2 \sin (u) \\
= & -\pi^{2}\left(1+t+t^{2}\right) \cos (\pi x) \\
& +(3+2 t)[1-\cos (\pi x)] \\
& +2 \sin \left(\left(1+t+t^{2}\right)(1-\cos (\pi x))\right), \\
& x \in(0,2), t>0,
\end{aligned}
$$

$u(x, 0)=1-\cos (\pi x)$,

$$
\left.\frac{\partial u}{\partial t}(x, t)\right|_{t=0}=1-\cos (\pi x)
$$$$
x \in(0,2) \text {, }
$$

$u(0, t)=u(2, t)=0, \quad t \geq 0$.

Its theoretical solution is $u(x, t)=\left(1+t+t^{2}\right)[1-\cos (\pi x)]$. We define

$$
\|e\|_{\mathscr{G}}=\|e\|_{l^{2}}=\left(\sum_{i=1}^{N} e_{i}^{2} h\right)^{1 / 2}
$$

where $e_{i}=u\left(x_{i}\right)-u_{h}\left(x_{i}\right)$ and the corresponding relative error is $\|e\|_{\mathscr{G}} /\left\|u_{h}(x)\right\|_{\mathscr{G}}$. The numerical results for the OSC scheme are given in Table 1 . In order to discuss the accuracy of the method at long time level, we give relative errors in the brackets. In [9], Cui approximates the second-order derivative in the space variable by compact finite difference. Table 2 gives error comparison of the Cui scheme [9] and the OSC scheme for $h=0.2$ and $h=0.05$ with $\tau=0.01$.

The rate of convergence of the proposed method can be calculated from the formula

$$
p=\frac{\log \left(\left\|u-u_{h_{1}}\right\|_{L^{2}} /\left\|u-u_{h_{2}}\right\|_{L^{2}}\right)}{\log \left(h_{1} / h_{2}\right)},
$$


TABLE 1: Errors of the OSC scheme for Example 1 with $\tau=0.01$.

\begin{tabular}{|c|c|c|c|c|}
\hline$\tau$ & $h=0.4$ & $h=0.2$ & $h=0.1$ & $h=0.05$ \\
\hline \multirow{2}{*}{1.0} & 0.005028 & $2.204825 e-04$ & $6.071307 e-05$ & $7.465548 e-05$ \\
\hline & $(4.628533 e-04)$ & $(2.028419 e-05)$ & $(5.585379 e-06)$ & $(6.868002 e-06)$ \\
\hline \multirow{2}{*}{2.0} & 0.018186 & $9.633026 e-04$ & $5.469951 e-05$ & $1.167707 e-04$ \\
\hline & $(7.193409 e-04)$ & $(3.808883 e-05)$ & $(2.162769 e-06)$ & $(4.617007 e-06)$ \\
\hline \multirow{2}{*}{3.0} & 0.038509 & 0.002078 & $4.165599 e-05$ & $8.650306 e-05$ \\
\hline & $(8.213101 e-04)$ & $(4.431546 e-05)$ & $(8.884192 e-07)$ & $(1.844895 e-06)$ \\
\hline \multirow{2}{*}{4.0} & 0.056416 & 0.003601 & $1.471193 e-04$ & $6.140355 e-05$ \\
\hline & $(7.459005 e-04)$ & $(4.760212 e-05)$ & $(1.944508 e-06)$ & $(8.115835 e-07)$ \\
\hline \multirow{2}{*}{5.0} & 0.091717 & 0.005319 & $2.522874 e-04$ & $6.190987 e-05$ \\
\hline & $(8.217853 e-04)$ & $(4.766007 e-05)$ & $(2.260542 e-06)$ & $(5.547237 e-07)$ \\
\hline$\vdots$ & $\vdots$ & $\vdots$ & $\vdots$ & $\vdots$ \\
\hline \multirow{2}{*}{10.0} & 0.356359 & 0.020142 & 0.001164 & $8.881136 e-06$ \\
\hline & $(8.931951 e-04)$ & $(5.048799 e-05)$ & $(2.910451 e-06)$ & $(2.226086 e-08)$ \\
\hline \multirow{2}{*}{11.0} & 0.421092 & 0.023767 & 0.001461 & $6.242861 e-06$ \\
\hline & $(8.811027 e-04)$ & $(4.972783 e-05)$ & $(2.995216 e-06)$ & $(1.306168 e-08)$ \\
\hline \multirow{2}{*}{12.0} & 0.485449 & 0.029326 & 0.001729 & $2.427837 e-05$ \\
\hline & $(8.606313 e-04)$ & $(5.198519 e-05)$ & $(3.064901 e-06)$ & $(4.303743 e-08)$ \\
\hline
\end{tabular}

TABLE 2: Relative errors comparison of the Cui scheme and the OSC scheme for Example 1 with $\tau=0.01$.

\begin{tabular}{|c|c|c|c|c|}
\hline \multirow{2}{*}{$t$} & $h=0.2$ & $h=0.2$ & $h=0.05$ & $h=0.05$ \\
\hline & The Cui scheme & The OSC scheme & The Cui scheme & The OSC scheme \\
\hline \multirow{2}{*}{1.0} & 0.0018 & $2.204825 e-04$ & $1.5975 e-04$ & $7.465548 e-05$ \\
\hline & $(3.3838 e-04)$ & $(2.028419 e-05)$ & $(3.0745 e-05)$ & $(6.868002 e-06)$ \\
\hline \multirow{2}{*}{2.0} & 0.0054 & $9.633026 e-04$ & $5.6131 e-05$ & $1.167707 e-04$ \\
\hline & $(4.4662 e-04)$ & $(3.808883 e-05)$ & $(4.6296 e-06)$ & $(4.617007 e-06)$ \\
\hline \multirow{2}{*}{3.0} & 0.0111 & 0.002078 & $2.4045 e-05$ & $8.650306 e-05$ \\
\hline & $(4.9330 e-04)$ & $(4.431546 e-05)$ & $(1.0679 e-06)$ & $(1.844895 e-06)$ \\
\hline \multirow{2}{*}{4.0} & 0.0194 & 0.003601 & $5.6697 e-05$ & $6.140355 e-05$ \\
\hline & $(5.3439 e-04)$ & $(4.760212 e-05)$ & $(1.5588 e-06)$ & $(8.115835 e-07)$ \\
\hline \multirow{2}{*}{5.0} & 0.0299 & 0.005319 & $1.3121 e-04$ & $6.190987 e-05$ \\
\hline & $(5.5700 e-04)$ & $(4.766007 e-05)$ & $(2.4436 e-06)$ & $(5.547237 e-07)$ \\
\hline$\vdots$ & $\vdots$ & $\vdots$ & $\vdots$ & $\vdots$ \\
\hline \multirow{2}{*}{10.0} & 0.1167 & 0.020142 & $4.5183 e-04$ & $8.881136 e-06$ \\
\hline & $(6.0677 e-04)$ & $(5.048799 e-05)$ & $(2.3501 e-06)$ & $(2.226086 e-08)$ \\
\hline \multirow{2}{*}{11.0} & 0.1413 & 0.023767 & $5.4285 e-04$ & $6.242861 e-06$ \\
\hline & $(6.1335 e-04)$ & $(4.972783 e-05)$ & $(2.3565 e-06)$ & $(1.306168 e-08)$ \\
\hline \multirow{2}{*}{12.0} & 0.1675 & 0.029326 & $6.4485 e-04$ & $2.427837 e-05$ \\
\hline & $(6.1597 e-04)$ & $(5.198519 e-05)$ & $(2.3714 e-06)$ & $(4.303743 e-08)$ \\
\hline
\end{tabular}

where $h_{1}, h_{2}$ are space steps and the value of $p$ is called the rate of convergence. In Theorem 6 , we prove that our proposed scheme is $\mathcal{O}\left(\tau^{2}+h^{4}\right)$. In Figure 1 , a comparison of the OSC scheme with the Cui scheme [9] has been made; the slope is 4 . When the space grid size $h$ is reduced by $1 / 2$ and the time grid size $\tau$ is reduced by $1 / 4$, the error between the analytic solution and the numerical solution is reduced by $1 / 16$. Thus the scheme is of fourth-order accuracy in space and second-order accuracy in time. From Figure 1, Tables 1 and 2, we can see that the OSC method is more efficient and accurate than the Cui scheme [9] though they have the same fourth order in space and second order in time, and the OSC method has conceptual simplicity. The space-time graphs of analytical and estimated functions are given in Figure 2 with $h=\tau=$ 0.01 . 
TABLE 3: Errors comparison of the Cui scheme and the OSC scheme for Neumann problem.

\begin{tabular}{|c|c|c|c|c|}
\hline$t$ & $\begin{array}{l}\quad h=0.02 \\
\text { The Cui scheme }\end{array}$ & $\begin{array}{c}\tau=0.02 \\
\text { The OSC scheme }\end{array}$ & $\begin{array}{c}\quad h=0.005 \\
\text { The Cui scheme }\end{array}$ & $\begin{array}{c}\tau=0.005 \\
\text { The OSC scheme }\end{array}$ \\
\hline \multirow{2}{*}{1.0} & 0.0121 & $2.118839 e-04$ & 0.0066 & $1.260930 e-05$ \\
\hline & $(0.0329)$ & $(5.875168 e-04)$ & $(0.0179)$ & $(3.444721 e-05)$ \\
\hline \multirow{2}{*}{2.0} & 0.0019 & $2.155475 e-04$ & 0.0014 & $1.347026 e-05$ \\
\hline & $(0.0141)$ & $(0.001625)$ & $(0.0103)$ & $(1.000319 e-04)$ \\
\hline \multirow{2}{*}{3.0} & 0.0016 & $6.177249 e-05$ & 0.0013 & $3.785799 e-06$ \\
\hline & $(0.0325)$ & $(0.001266)$ & $(0.0271)$ & $(7.642222 e-05)$ \\
\hline \multirow{2}{*}{4.0} & 0.0021 & $4.200750 e-05$ & $3.7009 e-04$ & $2.592393 e-06$ \\
\hline & $(0.1163)$ & $(0.002338)$ & $(0.0202)$ & $(1.422444 e-04)$ \\
\hline \multirow{2}{*}{5.0} & $8.0692 e-04$ & $2.568254 e-05$ & $1.2996 e-04$ & $1.588930 e-06$ \\
\hline & $(0.1198)$ & $(0.003886)$ & $(0.0193)$ & $(2.369950 e-04)$ \\
\hline$\vdots$ & $\vdots$ & $\vdots$ & $\vdots$ & $\vdots$ \\
\hline \multirow{2}{*}{10.0} & $2.4757 e-05$ & $1.744012 e-06$ & $4.2271 e-05$ & $1.057929 e-07$ \\
\hline & $(0.5453)$ & $(0.038895)$ & $(0.9311)$ & $(0.002340)$ \\
\hline \multirow{2}{*}{11.0} & $4.4015 e-05$ & $1.301622 e-06$ & $1.9435 e-05$ & $8.210182 e-08$ \\
\hline & $(2.6353)$ & $(0.079228)$ & $(1.1637)$ & $(0.004941)$ \\
\hline \multirow{2}{*}{12.0} & $3.2260 e-05$ & $1.014045 e-06$ & $5.5229 e-06$ & $6.173919 e-08$ \\
\hline & $(5.2505)$ & $(0.171864)$ & $(0.8989)$ & $(0.010115)$ \\
\hline
\end{tabular}

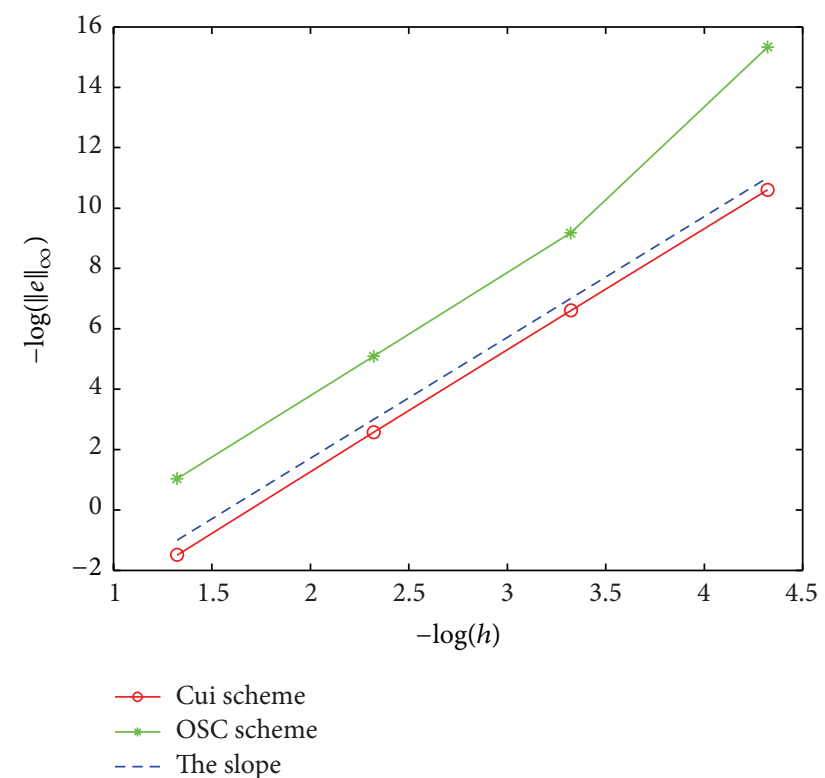

Figure 1: Convergence rate of two different schemes for Example 1 in $t=2.0$.

Example 2. We consider the problem with Neumann boundary conditions

$$
\begin{aligned}
& \frac{\partial^{2} u}{\partial t^{2}}+\frac{\partial u}{\partial t}-\frac{\partial^{2} u}{\partial x^{2}}+2 \sin (u) \\
& =\pi^{2} e^{-t} \sin (\pi x)+2 \sin \left(e^{-t} \sin (\pi x)\right), \\
& x \in(0,2), t>0, \\
& u(x, 0)=\sin (\pi x),
\end{aligned}
$$

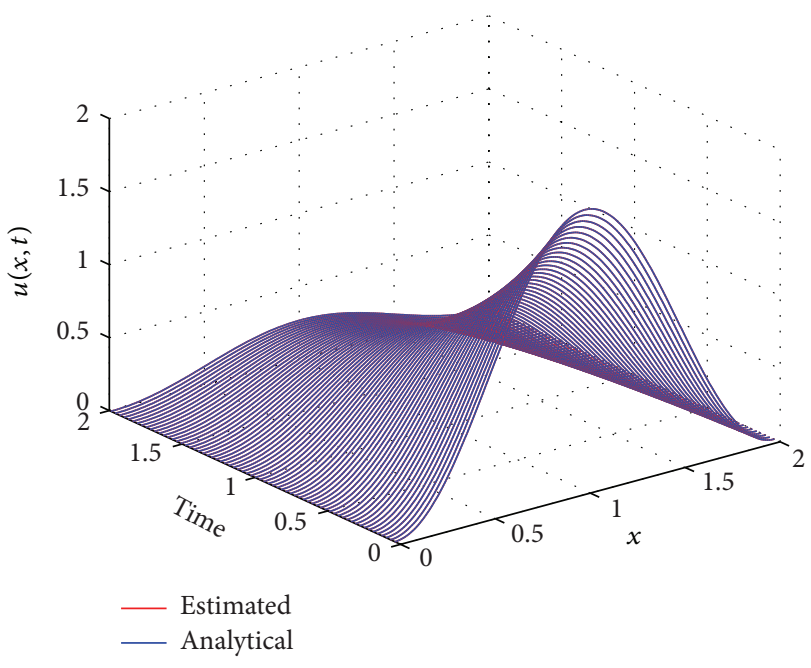

Figure 2: Space-time graph of the solution for Example 1 up to $t=$ 2.0 with $\tau=0.01$ and $h=0.01$, analytical solution with blue color and estimated solution with red color.

$$
\left.\frac{\partial u}{\partial t}(x, t)\right|_{t=0}=-\sin (\pi x)
$$$$
x \in[0,2],
$$$$
\frac{\partial u}{\partial x}(0, t)=\frac{\partial u}{\partial x}(2, t)=\pi e^{-t}, \quad t \geq 0
$$

Its theoretical solution is $u(x, t)=e^{-t} \sin (\pi x)$. Error comparison for $h=0.01$ and $\tau=0.01$ between the OSC scheme and the Cui scheme is given in Table 3. From Table 3, we can see that the OSC scheme is more accurate than the Cui 


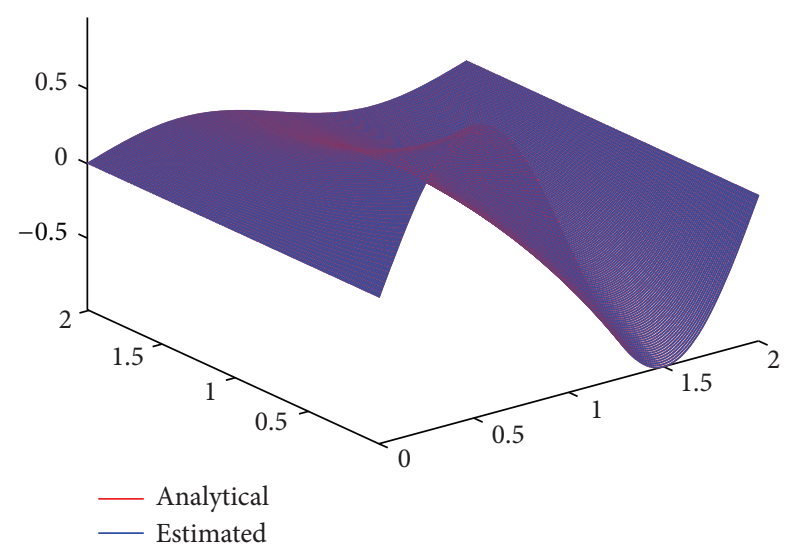

FIGURE 3: Theoretical solution (red color) and numerical solution (blue color) for Example 2.

scheme [9] according to their absolute error and relative error. The theoretical solution and the numerical solution $U_{j}^{n}$ with $h=\tau=0.01$ are plotted in Figure 3 .

\section{Conclusion}

In this paper, we discuss the generalized nonlinear sineGordon equation. We propose the OSC method to solve this nonlinear equation. The implementation of the method is as simple as finite difference methods. The numerical results given in the previous section demonstrate the accuracy of this scheme.

\section{Conflict of Interests}

The authors declare that there is no conflict of interests regarding the publication of this paper.

\section{Acknowledgments}

This work is partially supported by the National Natural Science Foundation of China under Grant 11271110, the Key Programs for Science and Technology of the Education Department of Henan Province under Grant 12A110007, and the Scientific Research Funds of Henan University of Science and Technology.

\section{References}

[1] P. J. Olver, Applications of Lie Groups to Differential Equations, vol. 107 of Graduate Texts in Mathematics, Springer, Berlin, Germany, 1986.

[2] M. J. Ablowitz and H. Segur, Solitons and the Inverse Scattering Transforms, SIAM, Philadelphia, Pa, USA, 1981.

[3] R. K. Dodd, J. C. Eilbeck, J. D. Gibbon, and H. C. Morris, Solitons in Nonlinear Wave Equations, Academic Press, New York, NY, USA, 1982.

[4] P. G. Drazin and R. S. Johnson, Solitons: An Introduction, Cambridge University Press, Cambridge, UK, 1989.
[5] A. Q. M. Khaliq, B. Abukhodair, Q. Sheng, and M. S. Ismail, "A predictor-corrector scheme for the sine-Gordon equation," Numerical Methods for Partial Differential Equations, vol. 16, no. 2, pp. 133-146, 2000.

[6] A. G. Bratsos, "A fourth order numerical scheme for the onedimensional sine-Gordon equation," International Journal of Computer Mathematics, vol. 85, no. 7, pp. 1083-1095, 2008.

[7] A. Mohebbi and M. Dehghan, "High-order solution of onedimensional sine-Gordon equation using compact finite difference and DIRKN methods," Mathematical and Computer Modelling, vol. 51, no. 5-6, pp. 537-549, 2010.

[8] Q. B. Xu and Q. S. Chang, "Two implicit difference schemes for the generalized nonlinear sine-Gordon equation," Acta Mathematicae Applicatae Sinica, vol. 30, no. 2, pp. 263-271, 2007 (Chinese).

[9] M. R. Cui, "Fourth-order compact scheme for the onedimensional Sine-Gordon equation," Numerical Methods for Partial Differential Equations, vol. 25, no. 3, pp. 685-711, 2009.

[10] B. Bialecki and G. Fairweather, "Orthogonal spline collocation methods for partial differential equations," Journal of Computational and Applied Mathematics, vol. 128, no. 1-2, pp. 55-82, 2001.

[11] J. Douglas Jr. and T. Dupont, Collocation Methods for Parabolic Equations in a Single Space Variable, vol. 385 of Lecture Notes in Mathematics, Springer, New York, NY, USA, 1974.

[12] M. P. Robinson and G. Fairweather, "Orthogonal spline collocation methods for Schrödinger-type equations in one space variable," Numerische Mathematik, vol. 68, no. 3, pp. 355-376, 1994.

[13] L. M. Zhang, "Convergence of a conservative difference scheme for a class of Klein-Gordon-Schrödinger equations in one space dimension," Applied Mathematics and Computation, vol. 163, no. 1, pp. 343-355, 2005.

[14] Q.-J. Meng, L.-P. Yin, X.-Q. Jin, and F.-L. Qiao, "Numerical solutions of coupled nonlinear Schrödinger equations by orthogonal spline collocation method," Communications in Computational Physics, vol. 12, no. 5, pp. 1392-1416, 2012.

[15] S.-W. Vong, Q.-J. Meng, and S.-L. Lei, "On a discrete-time collocation method for the nonlinear Schrödinger equation with wave operator," Numerical Methods for Partial Differential Equations, vol. 29, no. 2, pp. 693-705, 2013.

[16] Z. Fei and L. Vázquez, "Two energy conserving numerical schemes for the sine-Gordon equation," Applied Mathematics and Computation, vol. 45, no. 1, pp. 17-30, 1991.

[17] J. C. Díaz, G. Fairweather, and P. Keast, "Algorithm 603: COLROW and ARCECO: FORTRAN packages for solving certain almost block diagonal linear systems by modified alternate row and column elimination," ACM Transactions on Mathematical Software, vol. 9, no. 3, pp. 376-380, 1983. 


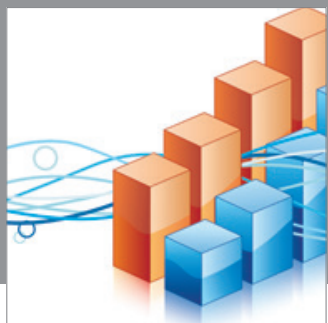

Advances in

Operations Research

mansans

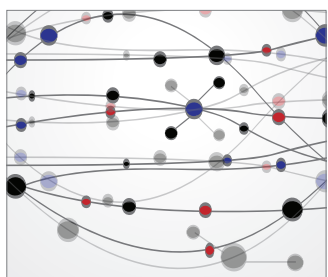

The Scientific World Journal
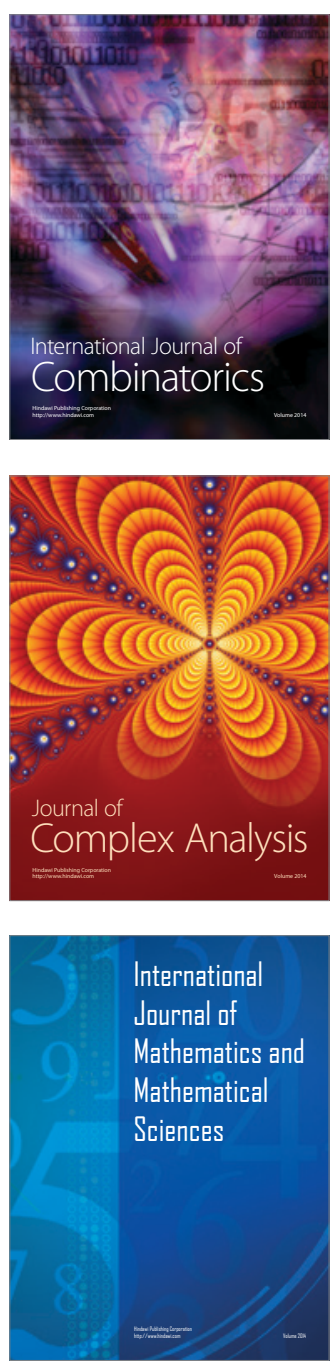
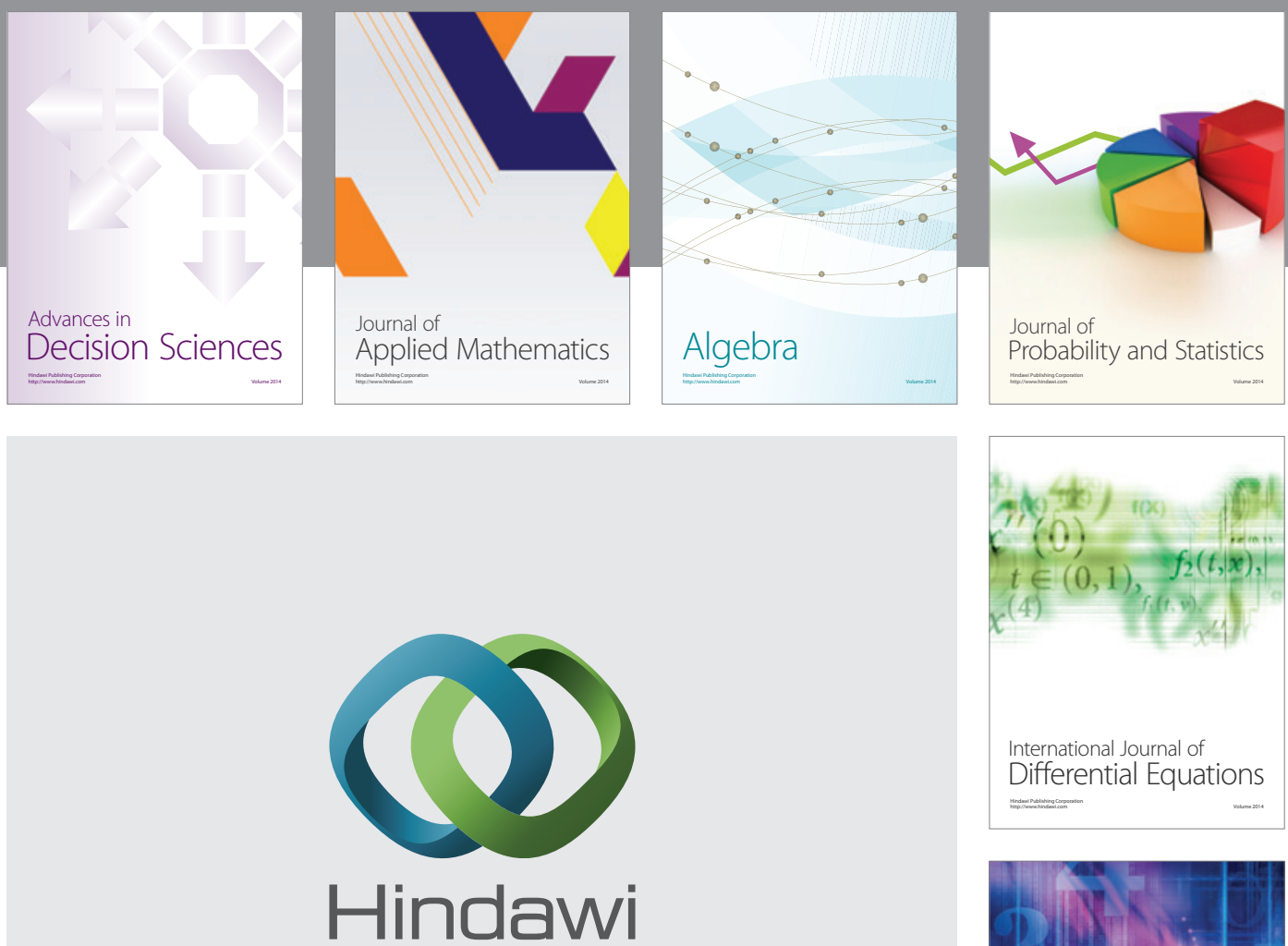

Submit your manuscripts at http://www.hindawi.com
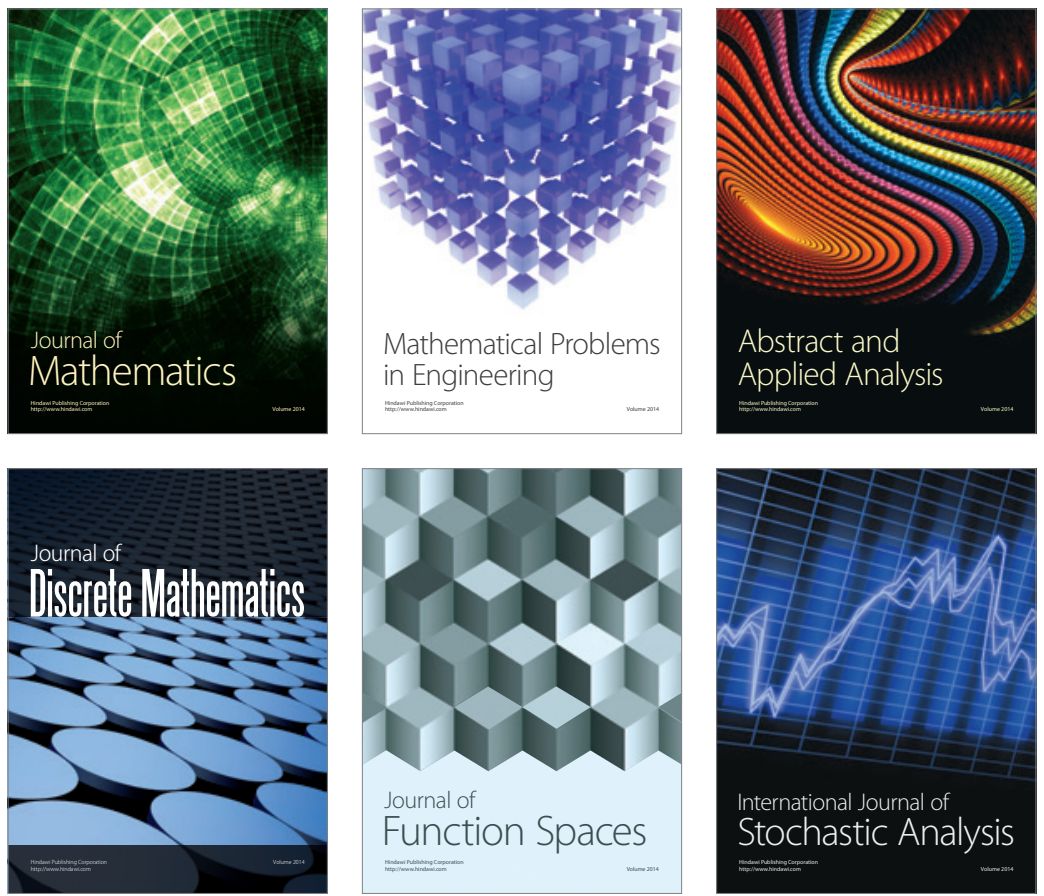

Journal of

Function Spaces

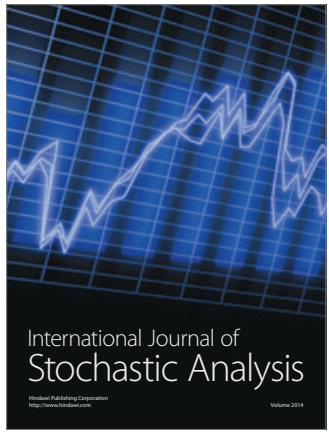

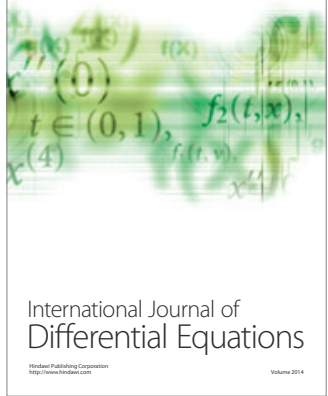
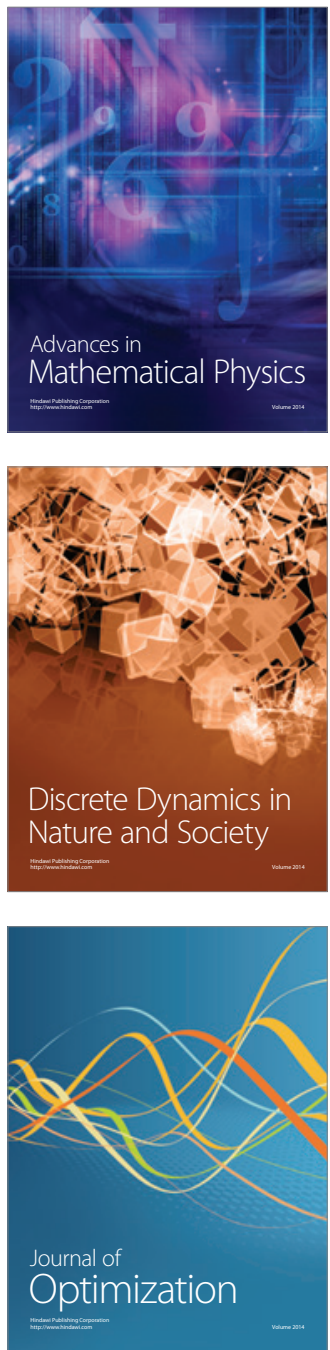